\title{
From Interference to Factitious Disorder: Slippery Slope or Tip of the Iceberg? The Case of a Difficult Guardian of a Head-Injured Adult
}

\author{
Bienca Lau
}

\begin{abstract}
Factitious disorder imposed on another is predominantly recognized in the pediatric population. It represents a diagnostic challenge and can have a profoundly negative influence on the illness and management of adult patients. This report describes a case of factitious disorder imposed on an adult with acquired brain injury and discusses the diagnostic and management challenges. Positive outcome followed appropriate limit-setting.
\end{abstract}

Keywords: Factitious disorder; Factitious disorder imposed on another; Munchausen syndrome; Munchausen syndrome by proxy; Head injury; Surrogate decision maker; Splitting; Unexplained medical symptom

\section{Introduction}

While one recognizes that supportive relationships are beneficial [1-5], the experience of such relationships is subjective. Apparent supportive relationships can also have a negative effect. When a patient cannot provide informed consent, the involvement of support person(s), particularly the surrogate decision-maker, becomes critical. At times, surrogates may not protect the patient's autonomy when making medical decisions due to various reasons [6]. The situation is even more complex when the surrogate has priorities other than the best outcome for the patient. When a patient's longitudinal course appears atypical, factitious disorder (FD) and factitious disorder imposed on another (FDIA) should be considered in the differential diagnosis. In FDIA, caregivers fabricate or induce signs and symptoms in a dependent person, meeting their own psychological needs through the attention from medical personnel caring for their dependent, most of whom are children but rarely, can be compromised adults [7, 8] like Mr. A.

Manuscript submitted March 4, 2018, accepted March 15, 2018

Department of Psychiatry, University of Saskatchewan, 103 Hospital Dr., Saskatoon, Saskatchewan, SK S7N 0W8, Canada.

Email: Bienca.lau@saskhealthauthority.ca

doi: https://doi.org/10.14740/jmc3030w

\section{Case Report}

Mr. A was a manual worker in his 50 s at the time of his accident. His long-time common-law partner was Ms. B, also in her 50s. Neither of them had children. There was no psychiatric history prior to his head injury, which resulted in a left subdural hematoma. After his 7-month admission to an acute facility, Mr. A lived in a series of personal care homes. His challenges included cognitive impairment, mood lability, disinhibition, perseveration and aggression. Eventually, after a psychiatry unit admission lasting several months, he was placed in long-term care.

As Mr. A's guardian, Ms. B met with Dr. C during the initial consultation at the long-term care facility. Mr. A had just started treatment with carbamazepine. Ms. B insisted that carbamazepine should be discontinued if she was not satisfied within 6 weeks. Since the serum level of carbamazepine was subtherapeutic, an increase in the dose was suggested in two increments, testing the level 1 month afterwards. Ms. B demanded the second increment take place only after the level was obtained. Paradoxically, she also challenged the use of carbamazepine level in clinical decision-making. During this meeting, Ms. B sat unnecessarily close to Dr. C. She was confrontational. Toward the psychiatry resident, she remarked, "So, you're tagging along?" Alternatively, when her requests were accommodated, she became over-familiar. Thereafter, Ms. B frequently requested meeting with Dr. C and regularly questioned the level of carbamazepine.

Three months later, Ms. B requested replacing as needed haloperidol with lorazepam. Dr. C explained that benzodiazepines can disinhibit head-injured adults. Ms. B persisted and ultimately, Dr. C agreed to a 2-week trial, hoping to gain Ms. B's trust. In the next 3 days, Mr. A's outbursts soared. Ms. B consented to stopping lorazepam. Ten days later, Ms. B demanded a retrial, which Dr. C declined. Dr. C expressed concerns about Ms. B's behaviour to the unit manager, who responded that Ms. B had Mr. A's "best interest at heart".

Despite steady increments, carbamazepine remained subtherapeutic, even with the auto-induction properties of carbamazepine taken into consideration. Ms. B wanted it discontinued because she claimed carbamazepine caused Mr. A's pain, which never resulted in behaviour observable to the staff.

Ms. B had idiosyncratic beliefs about various metabolic issues. For example, she insisted that Mr. A consume eight to 10 glasses of water around each meal to treat dehydration, de- 
spite the lack of clinical evidence of dehydration in Mr. A. In fact, his blood tests showed hyponatremia (that was later concluded to be the consequence of excessive water intake). She also demanded Dr. C to amend entries in Mr. A's chart that related to her requests of medications for him. After further incidents at the long-term care facility, Dr. C documented that Ms. B's interference could "seriously impact clinical decisions". This was noted by Ms. B during her weekly review of Mr. A's chart. She immediately terminated Dr. C. The unit manager asked Dr. C to "try working with Ms. B." Unbeknown to Dr. $\mathrm{C}$, another unit manager had written to Ms. B regarding boundary issues with other residents at the facility. Management then sought legal counsel, who informed Ms. B of the need to follow medical recommendations. Concerns about her capacity to be Mr. A's guardian were dismissed by management. Ms. B disregarded the lawyer's letter and started to openly interfere with the administration of carbamazepine. She drew staff into protracted and counterproductive conversations. Ultimately, Dr. C contacted the Canadian Medical Protection Association. The medical officer of the association suggested that the health team had the duty to bring the matter to the attention of the public trustee. Subsequently, management became more receptive. The team decided to challenge Mr. B's status as the guardian. Mr. A's hospital charts were reviewed in preparation of the affidavit. In addition to a similar pattern of interference from Ms. B in Mr. A's previous hospitalization, the review also uncovered a documented incident of her administering an unknown substance to Mr. A.

\section{Discussion}

Establishing the diagnosis of FDIA is difficult. Perpetrators conceal their activities. Once these activities are uncovered, it is seldom easy to ascertain intentions. In this case, Mr. A had a clearly established diagnosis of acquired brain injury, with cognitive impairment and behavioural disturbance. It was difficult to see which part of Mr. A's behavioural symptoms was due to his head injury and which were the consequences of Ms. B's withholding his medications, her exaggeration or misreporting, his hyponatremia due to the polydipsia forced by Ms. $\mathrm{B}$, and even her covert administration of unknown substances. Ms. B's presentation was dominated by her exaggerated concerns and boundary issues. The deceptive component, essential for the diagnosis of FDIA, was subtle. The possibility that she had been knowingly withholding Mr. A's carbamazepine for months, while constantly questioning its level, only surfaced after she openly refused to let Mr. A take the medication. Other examples of deception involved more fabrication than induction. Her excessive demand of attention from medical staff and her insatiable need to be involved in medical minutia are commonly observed in FD and FDIA.

Rigour is critical in distinguishing intentional interference of a caregiver from alternative explanations. Once diagnosed, the treatment team initiates dialogue with the perpetrator, who often disengages him- or herself and the victim from the institution, and begins dealing with new and unsuspecting care providers, similar to cases of factitious disorder imposed on self [9]. Therefore, when the team decided to challenge Ms. B's guardian status, restrictions were immediately placed on her visitations, even before the court application was filed. In contrast to her usual demand to meet with team members, Ms. $\mathrm{B}$ refused to attend the meeting scheduled by the team to discuss her guardian status, once she realized that legal counsel would be present.

Aside from Ms. B's unhelpful behaviour, management's approach perpetuated the situation. It is disconcerting for healthcare providers to see patients' family as ill-willed. It was counterintuitive to view Mr. A, a tall and muscular male aggressor, as a victim. Contemporaneously, staff had been under stress because of other residents who were lodging unfounded complaints. This further promoted management's conflict avoidance. Addressing the interference of Ms. B required a concerted approach from the team. In this case, simply informing the management about FDIA and the potential outcome did not control the splitting, as they had been driven by fear of litigation. Strategically, consultation with the medical protection association provided the necessary medicolegal base for management to confront Ms. B. No less importantly, the medical protection association also counselled Dr. C on the potential of legal action from Ms. B against Dr. C and offered further support in the future.

Suspension of Ms. B's guardian status was granted by the public trustee. Restrictions to Ms. B's visitations were maintained, specifically to stop the interference around medications and excessive water consumption. With the lack of willing family members to assume the surrogacy role, medical decisions were to be made by agreement between two physicians, under the Substitute Health Care Decision Makers Act. Ms. B's interactions with other residents were monitored as well. Improvement of Mr. A's mental state soon followed. Verbal aggression, physical aggression, and perseverations decreased by $75 \%$. Staff ensured his compliance and his carbamazepine level became therapeutic. His hyponatremia normalized. Notwithstanding, Ms. B still triangulated a family physician from outside the facility into challenging Mr. A's fluid requirements. Notably, the family physician displayed characteristics of the likely physician in the patient-perpetrator-physician triad in Squires's review [10]. He stated that, in his experience with institutions, "things are not always done properly". He favored other explanations for Mr. A's hyponatremia. He acknowledged having 3-h counseling sessions with Ms. B. Later, Ms. B reported that Mr. A had flank pain due to dehydration. The concern was investigated and proven unfound.

\section{Conclusions}

FDIA can have profound impact on the presentation and management of adult patients who are cognitive impaired or otherwise incapable of advocating for themselves. When the clinical presentation is atypical, FDIA should be considered in the differential diagnoses. Familiarity with FDIA, careful documentation not only of the patient's state but also the behaviour of support persons, and comprehensive chart reviews can help establish the diagnosis. Physician and systems factors can ex- 
acerbate or mitigate the impact of FDIA. Protecting the interests of the compromised patient is essential, and may require challenging the established surrogacy status and minimizing the potential of elopement or sabotage. Medicolegal counsel is invaluable. Gains can be achieved and sustained with appropriate limit-setting.

\section{Acknowledgments}

BL is grateful to Dr. Rudy Bowen and Dr. Mansfield Mela for valuable critique of the manuscript, and to Dr. Lloyd Balbuena for technical assistance.

\section{Grant Support}

None.

\section{Conflict of Interest}

None.

\section{References}

1. Choenarom C, Williams RA, Hagerty BM. The role of sense of belonging and social support on stress and de- pression in individuals with depression. Arch Psychiatr Nurs. 2005;19(1):18-29.

2. Cobb S. Presidential Address-1976. Social support as a moderator of life stress. Psychosom Med. 1976;38(5):300314.

3. Lackner JM, Gudleski GD, Firth R, Keefer L, Brenner DM, Guy K, Simonetti C, et al. Negative aspects of close relationships are more strongly associated than supportive personal relationships with illness burden of irritable bowel syndrome. J Psychosom Res. 2013;74(6):493-500.

4. Ross CE, Mirowsky J. Family relationships, social support and subjective life expectancy. J Health Soc Behav. 2002;43(4):469-489.

5. Thoits PA. Mechanisms linking social ties and support to physical and mental health. J Health Soc Behav. 2011;52(2):145-161.

6. Pope TM. Legal fundamentals of surrogate decision making. Chest. 2012;141(4):1074-1081.

7. Deimel GWt, Burton MC, Raza SS, Lehman JS, Lapid MI, Bostwick JM. Munchausen syndrome by proxy: an adult dyad. Psychosomatics. 2012;53(3):294-299.

8. Burton MC, Warren MB, Lapid MI, Bostwick JM. Munchausen syndrome by adult proxy: a review of the literature. J Hosp Med. 2015;10(1):32-35.

9. Chapman JS. Peregrinating problem patients; Munchausen's syndrome. J Am Med Assoc. 1957;165(8):927-933.

10. Squires JE, Squires RH, Jr. Munchausen syndrome by proxy: ongoing clinical challenges. J Pediatr Gastroenterol Nutr. 2010;51(3):248-253. 